\title{
अरुणकमल की कविताओं में अंतरराष्ट्रीय चेतना
}

\author{
Guhanandan
}

Research Scholar, Hindi \& Sanskrit Teacher, BVMGLOBAL Senior Secondary School, Morais City, Guntoor, Trichy, Tamilnadu

शोध-लेख - अरुणकमल सत्तरोत्तर कवियों में प्रमुख और विशष्ट हैं । अंग्रेजी के प्राध्यापक होते हुए भी हिन्दी कविता में उनकी विशेष रुचि व आसक्ति बनी रही। समसामयिक मुद्दों को केंद्रित करके उन्होंने अपनी कविताओं का सृजन किया है। साम्यवादी विचारधारा परिपर्ण प्रगतिवादी काव्य की विशेषताओं से अपने काव्य को समलंकृत करते हुए समसामयिक तत्वों को अपने काव्य का विषय बनाकर अपनी केवल धार से लेकर योगफल तक काव्य संग्रहों का प्रकाशन करवाया । उनका एक अनूदित काव्यसंग्रह जब पुकारती है कोयल है जोकि वियतनामी कवि 'तो हू ' की प्रगतिवादी उद्रार व भावाभिव्यंजना थी। कवि की चेतना स्थानीय समसामयिक मुद्दों को विचार प्रदान करने से एक कदम आगे अंतरराष्ट्रीय मुद्दों को अपनी वाणी देना चाहती है । यह उनके अंतरराष्ट्रीय नागरिकता का परिचायक है । कवि अरुणकमल ने प्रगतिवादी कविता व समसामयिक कविता से अंतरराष्ट्रीय विचारधारा को निरूपित किया है। इस शोध-लेख का उद्देश्य है कि अरुणकमल की कविताओं में होनेवाली अंतरराष्ट्रीय चेतना प्रतिपादित हो ।

मुख्य शब्द - आयाम; चेतना; विश्वप्रजापतित्व; अपमिश्रण; भौगोलीकरण; अवगमन; संवेदनशीलता ।

\section{1. चेतना व उसके आयाम}

“सामान्यतः चेतना से तात्पर्य हम किसी देश, काल से संबंधित मानव समाज में अभिव्यक्त जागृति को समझते हैं । जागृति प्रतिक्रियात्मक होती है । चेतना तत्कालीन जीवन में गतिरोध एवं गतिशीलता से उत्पत्र हो सकती है। इसकेलिए सामाजिक, आर्थिक, सांस्कृतिक स्थितियाँ प्रेरणादायी हो सकती हैं |“ [1]

चेतना अवगमन की वस्तु है जिसे पारिभाषित करना आसान नहीं है। व्यक्ति चेतना से ही सक्रिय होता है। चेतना का रूप अत्यन्त सूक्ष्म और जटिल है। इसकी व्याख्या नियंत्रित शब्दों में नहीं की जा सकती है। फिर भी विचारकों ने अपने-अपने दृ्टिकोण से चेतना की व्याख्या करने का प्रयास किया है। 'चेतना' शब्द ‘चित' से सम्बन्धित है। संस्कृत आचार्यों ने चेतना को बुद्धिज्ञान, जीवन-शक्ति, भावना या विचार के अर्थ में ग्रहण किया है। धी:, मति, चित, सवित्त, प्रतिपत, ज्ञाप्ति, सत्व एवं जीवतं के अर्थ में भी चेतना शब्द का प्रयोग किया जाता है।

'चैतन्य लक्षणा ‘जीव:' अथार्त् जीवन का लक्षण ही चेतना है। चेतना का कोशगत अर्थ है - चैतन्य, ज्ञान, होष, याद, बुद्धिगत, जीवन शक्ति आदि। पं. रामचन्द्र वर्मा अपने मानक हिंदी कोश में चेतना के विषय में लिखते हैं - चेतना जीव या प्राणी के अन्तर्बाह्य तत्वों या बातों का अनुभव या मान करती है। इस प्रकार चेतना मन की एक स्थिति ही है - जिसके अन्तर्गत बाह्य जगत के प्रति संवदेनशीलता तीव्र अनुभूति का आवेग, चयन या निर्माण की शक्ति इन सबके प्रति चिन्तन विद्यमान रहता है। ये सब बातें मिलकर किसी भी व्यक्ति की पूर्ण चैतन्य अवस्था का निर्माण करती है। स्पष्ट है कि चेतना के बिना मनुष्य जीवित नहीं बल्कि मृत के समान है। चेतना से ही हमारा जीवन गति व विकास करने की शक्ति लेता है। चेतना जागरूकता लाने और सकंल्प लेने में अहम भूमिका निभाती है जिससे कार्य सम्पत्र हाते हैं। 'चेतना'
शब्द का प्रयोग प्राय: मनोवैज्ञानिक तथा दार्शनिक अर्थों मे ही किया जाता है। चेतना मानव की प्रमुख विशेषता है जिसे वस्तुओं, विषयों, व्यवहारों का ज्ञान भी कहा जा सकता है। मनोवैज्ञानिक अर्थ मे - 'चेतना सभी प्रकार के अनुभवों का संग्रहालय है।' कह सकते हैं कि चेतना व्यक्ति की वह केन्द्रीय शक्ति है जो अनुभूति, विचार, चिन्तन, संकल्प, कल्पना आदि क्रियाएँ करती है। यदि चेतना को अलग कर दे तो दुनिया में सब कुछ होगा, सिर्फ समय नहीं होगा। समझा ले कि इस पहाड पर का चेतना नहीं है तो पत्थर होंगे, पहाड़ होगा, चाँद निकलेगा, दिन डूबेगा लेकिन समय जैसी कोई चीज नहीं होगी क्योंकि समय का बोध ही चेतना है। चेतना और मनुष्य का मौलिक संबंध है। चेतना वह विशेष गुण है जो मनुष्य को सजीव बनाती है आरै चरित्र उसका वह संपूर्ण संगठन है। किसी मनुष्य की चेतना और चरित्र केवल उसी की व्यक्तिगत संपत्ति नहीं होते। ये बहुत दिनों के सामाजिक प्रक्रिया के परिणाम होते हैं। प्रत्येक मनुष्य स्वयं के वंशानुक्रम प्रस्तुत करता है। वह विशेष प्रकार के संस्कार पतैक सम्पत्ति के रूप में पाता है। वह इतिहास को भी स्वयं में निरूपित करता है क्योंकि उसने विभित्र प्रकार की शिक्षा तथा प्रशिक्षण को जीवन में पाया है। इसके अतिरिक्त वह दूसरे लोगों को भी अपने द्वारा निरूपित करता है, क्योंकि उसका प्रभाव उसके जीवन पर उनके उदाहरण, उपदेश तथा अवपीड़न के द्वारा पडा है। एक बार मनुष्य की चेतना विकसित हो जाती है, तब उसकी प्राकृतिक स्वतन्त्रता चली जाती है। वह ऐसी अवस्था में विभित्र प्रेरणाओं और भीतरी प्रवृत्तियों से प्रेरित होता, परन्तु वह उन्हें स्वतन्त्रता से प्रकाशित नहीं कर सकता। इस पक्रार मनुष्य की चेतना अथवा विवेकी मन उसके अवचेतन अथवा प्राकृतिक मन पर अपना नियंत्रण रखता है। मनुष्य और पशु मे यही विशेष भेद है। मनुष्य की चेतना जागृत होती है परन्तु पशु की नहीं। चेतना का हमारी जीवन-शैली में बहुत महत्त्व है। मनोविज्ञान की हृष्टि में चेतना मानव में उपस्थित वह तत्त्व है जिसके कारण उसे सभी प्रकार की अनुभूतियाँ होती हैं। चेतना के कारण ही हम देखते, सुनते, समझते और अनेक विषयों पर चिंतन करते हैं। इसी के कारण हमें सुख-दु:ख की अनुभूति हाती है। मानव चेतना की तीन 
विशेषताएँ हैं। वे ज्ञानात्मक, भावात्मक और क्रियात्मक हाती हैं। चेतना ही सभी पदार्थों की जड़-चतेन, शरीर-मन, निर्जीव-सजीव, मस्तिष्क-स्रायु आदि को बनाती है। उनका रूप निरूपित करती है। चेतना के विषय में हम स्पष्ट रूप से कह सकते हैं कि मनुष्य के मस्तिष्क में हाने वाली क्रियाओं अर्थात कुछ नाडियों के स्पंदन का परिणाम ही चेतना है। यह अपने में स्वतन्त्र कोई अन्य तत्व नहीं है। शरीर चेतना के कार्य करने का यंत्र मात्र है, जिसे वह कभी उपयोग में लाती है और कभी नहीं लाती है। परन्तु यदि यंत्र बिगड़ जाए या टूट जाए तो चेतना अपने कामों के लिए अपंग हो जाती है। चेतना के बिना सुना व देखा नहीं जा सकता है। यह हमें अनुभव और भावनाओं की पहचान करने की अनुमति देता है। इस प्रकार चेतना मानसिक अनुभूति है। चेतना के विभिन्न आयाम होते हैं । वे हैं सांस्कृतिक आयाम, सामाजिक आयाम, आर्थिक आयाम, राजनीतिक आयाम, पर्यावरणीय, स्त्री-संबंधी आयाम, अंतरराष्ट्रीय आयाम आदि । हर कवि के काव्य के विभिन्न पहलुओं को खोजने का प्रयास शोध-विषय हो सकता है ।

\section{2. अंतरराष्ट्रीय चेतना और उसके आयाम}

चेतना समझने की प्रक्रिया है । राष्ट्रीय चेतना अमुक राष्ट्र के संपूर्ण पहलुओं, हर क्षेत्र-विशेष के प्रति जीनकारियों, समस्याओं आदि से अवगत होकर अपनी अनुभूति की भावाभिव्यंजना करना होता है । उसमें कलाकार के सांस्कृतिक, दार्शनिक, सामाजिक, राजनीतिक, नारीपरक, पर्यावरण-प्रदूषण, जलवायु परिवर्तण आदि आधुनिक समस्याओं के विचार विद्यमान होते हैं।

सार्वभौमिक चेतना वह क्षमता है जिससे चेतनायुक्त व्यक्ति सार्वभौमिक, अंतरराष्ट्रीय और विभित्र संस्कृतियों पार स्रोतों को समझता है । विभिन्न प्रणालियों, पद्धतियों, संस्थाओं, घटनाओं, संक्रियाओं के प्रभाव व अंतर्संबंध को समझ सकता है । अन्य संस्कृतियों की न्यायसंगति को मूल्यांकित व स्वीकृत करने में सामर्थ्यवान होता है । ऐसी चेतना से वह खुले दिल से प्रभावोत्पादक ढंग से उनजगहों में क्रियाशील होता है, जहाँ उसकी भाषा व संस्कृति नहीं है । दुनिया के हर राष्ट्र में कुप्रचलित मुद्दों व मसलों को वाणी देना चाहता है और वह अपेक्षा करता है कि ऐसी सामाजिक सिद्धांतों व विभित्र वादों, सामाजिक समस्याएँ जैसे मूलभूत मानव-अधिकार, सामाजिक न्याय आदि से हर देश की प्रजा अवगत हो जाए। इस तरह सामाजिक समस्याओं और विविध विषमताओं के प्रति जागरूक होकर तत्संबंधी अंतरराष्ट्रीय व्यवहार प्रकट करके समता व तालमेल स्थापित करने की उम्मीद रखता है।

सार्वभौमिक चेतना सार्वभौमिक समस्याओं को समझकर सुलझाने की क्रियाशीलता या प्रगतिशीलता है । समसामयिक जगत के धरातल से संसार के अन्यों और स्वयं को अवगत होने की प्रकृति व तत्परता है । स्थानीय समस्याओं को अंतरराष्ट्रीय हृष्टिकोण से सुलझाने का तरीका अपनाना और अंतरराष्ट्रीय मुद्धों को अपनी बुद्धिविचार से प्रभावित करना दोनों प्रासंगिक मान्यता है। किसी भी अंतरराष्ट्रीय समस्या के कई आयाम हो सकते हैं
जिनमें सार्वभौमिक संवेदनशीलता, सार्वभौमिक अवगमन की क्षमता और सार्वभौमिक स्वभागिता प्रमुख है । समाज के हर व्यवहार व घटना के प्रति सार्वभौमिकता की दृष्टिकोण से संवेदनशील होकर उसको सुधारने की कोशिश करना है । जगत की हर प्रभावी मुद्दों के प्रति जानकारी रखना जगत की प्रगति की तत्परता है । हर समस्या का अवगमन उसके समाधान की प्रथम प्रक्रिया है । समस्या के हल समझौतात्मक व्यवहार से स्थानीय और अंतरराष्ट्रीय गतिविधियों के आधार पर हो । हर अंतरराष्ट्रीय मद्दे को सार्वभौमिक प्रजा के नजरिये से देखना व परखना सार्वभौमिक समस्याओं की स्वभागिता है। कवि अपनी अंतरराष्ट्रीय चेतना से सार्वभौमिक प्रगतिशीलता व विश्व में स्वभागिता को निरूपित करता है ।

\section{3. कवि अरुणकमल की कविता में अंतरराष्ट्रीय चेतना}

देश-विदेश की समस्या है मिलावट या अपमिश्रण, चाहे देश विकसित हो या विकासशील देश हो । खाद्यपदार्थों में अनैतिक रूप से मूल्यहीन पदार्थों को मिलाकर आमदनी में वृद्धि करने का अन्याय बरसों से कुप्रचलित हरकत वाणिज्य के क्षेत्र में देखा जा रहा है । जनता के स्वास्थ्य पर उसका असर विभित्र रोग व रोगों के लक्षण, आवश्यक व अपेक्षित पौष्टिक तत्व उन अपमिश्रित खाद्यों में न होने के कारण हैं । धन-लोलुप व्यापारी जनता की सेहत का फिक्र नहीं करते । विटामिनों का भंडार है फल । उनको अप्राकृतिक ढंग से पकाना अनैतिक, अवैध और अस्वस्थ व्यवहार जिससे अतिसार, वमन, अंधापन, पेट-दर्द, अल्सर इत्यादि बीमारियाँ उत्पन्न होती हैं और वही चिरकारी रोगों में परिवर्तित होकर घातक बन जाती हैं। रासायनिक पदार्थ जैसे कारबाइड-पत्थर, ईथिलीन आदि के प्रयोग से फलों को असमय में परिपक्र करना भी अपमिश्रण के तहत माना जाता है । असमय एवं अप्राकृतिक पकाए गए फलों में अपेक्षित सूक्ष्मपौष्टिकतत्व, कनिज-पदार्थ, अनिवार्य आम्ल-तत्व, विटामीन आदि के न होने से सेहत पर भारी पडता है और इसके प्रतिकूल अपमिश्रित फल हानिकारक बन जाते हैं। देश-विदेश के शासन तंत्र ऐसी व्यापारी धूर्तता पर प्रतिबंध लगा चुका है । एक ओर विषैली वायु से असमय में यानी तुरन्त फलों को पकाने की चेष्टा है, तो दूसरी ओर फलों को देरी पकाने की हरकत यानी वणिक जब चाहते हैं और जब उन फलों की मांग हो, तब चालाकी से पकाना तो व्यापारी धूर्तता की पराकाष्ठा है। इसलिए तो कवि पेड-पौधे पर ही पकनेवाले फल की सुंदरता पर मुग्ध है । उनको शुक्र है कि जिन्होंने पेड पर ही फल को पकने दिया । ऐसा फल स्वास्य्यवर्धक है। प्राकृतिक पौष्टक पदार्थों से लदा होता है। कवि का मन इन विचारों से आह्नादित हो जाता है । अतः उन्होंने 'अपनी केवल धार' काव्यसंग्रह की ऐसी एक शोभनीय कविता का अनायास सृजन किया, जिसका शीर्षक है 'जाना है'।

“पहले भी देखा था यह फल सूँघा था 
चखा था बहुत बार

बचपन से ही

पर आज पहली बार जब देखा है

डाल पर पकते इस फल को

तभी जाना है असली रंग-स्वाद-गन्ध

इस छोटे-से फल के

धरती-आकाश तक फैले संबंध।"

कवि अरुणकमल जी विश्व के समाजवादी देश वियतनाम की राजनीतिक, सामाजिक और आर्थिक घटनाओं से अवगत हैं । वे वियतनाम जैसे कम आबादीवाले देशों से प्रेरित और उत्साहित हैं। अपने हुसूलों के लिए डटकर लडने की शक्ति व स्फूर्ति वियतनाम व काम्भोज देशों में भरमार है । वियतनाम आततायीशक्ति का परिचायक है। कोई लघु देश अपनी सैनिक शक्ति के बल पर 1954 से लेकर 1973 तक समर के क्षेत्र पर अडिग रहे, जबकि कोई आजकल के आधुनिक अस्त्र नहीं थे । वियतनाम में आंतरिक युद्ध भी जारी रहे । काम्भोज साम्राज्य तो उससे भी लघु देश है, जो वियतनाम पर नदी के लिए वार करता था। इनसब के हालात की पृष्ठभूमि पर लिखी गई कविता है 'मई का एक दिन'।
“वह कोई दिन था मई के महीने का
जब वियतनाम सीढ़ियों पर बैठा
पोंछ रहा था
खून और घावों से पटा शरीर
कम्बोडिया जकडी सिकड़ियाँ खोलता
गृह-प्रवेश की तैयारियों में व्यस्त था
और नीला आकाश ताल ताल में
फेंक रहा था अपनी शाखें ।" [3]

कवि वियतनाम और काम्भोज को ताकत और हिम्मत के प्रतीक मानते हैं। जब मानव को नैराश्य घेर लेता है, तब स्पूर्ति व प्रेरणा से ढाढस बंधाने का पवित्र कार्य दैवी स्वीकृत किया गया है । कवि को वियतनाम व काम्भोज की डटकर लडने की शक्ति और अपने अधिकारों के लिए हुंकार चाहे दुनिया के किसी भी कोने मिले श्लाघ्य है । ये दोनों देश कई विदेशी आक्रमणों से आक्रांतित थे, आपसी समस्याओं के लिए झूझ रहे थे और कई उपनिवेशी ताकतों से दबाए गए फिर भी उन के मन की शक्ति व आशा रही कि वे फिर से उभर उठेंगे, शोभायमान स्वशासन करेंगे और अपने देश का स्व-स्वसिद्धांतोंवाला पुनर्निमाण होगा । इसकी तारीफ में कवि आशावादी नजर आ रहे हैं।

“ऐसा ही दिन था वह मई के महीने का जब भविष्य की तेज धाह मेरे चेहरे को तृप्त कर रही थी -

तुमने, वियतनाम, तुमने मुझे दी थी वह ताकत कम्बोडिया, तुमने, तुमने मुझे दी थी वह हिम्मत

कि मैं भविष्य से कुछ बातें करता

टहल रहा था -

क्या हुआ जो मैं बहुत हारा था
बहुत खोया था

और मेरा परिवार तकलीफों में गर्क था

जब तुम जीते तब मैं भी जीता था।" [4]

संसार के सभी राष्ट् इस यांत्रिक युग के भागीदार हैं। वे एक दूसरे के पूरक एवं अन्योन्याश्रित हैं। हमारी संस्कृति की विशेषता है कि सारी भूमि को एक परिवार मानें । हमें किसी के आश्रय की जरूरत न रही। दुनिया की हर विविधता भारत में है, फिर भी ऐसी राय ऊँचे दर्जे की है । हमारे ऋषि-मुनियों की दूरहष्टि और वसुधैवकुटुम्बकम् की भावना दौडधूप की भौगोलीकृत दुनिया में साकार है। विकसित देशों के बुद्धिमानों ने इस धरती को 'ग्लोबल विल्लेज' माना है । कवि ने अपनी कविता 'जितना दो आँखें' में इस दृष्टि को ख्याल में “धरते-धरते लिखा है,
"किसी दिन तो कभी तो
बढ़ी होंगी जड़ें अन्दर
और चलकर पिया होगा
एक ही जल-कुण्ड से
जल
कहीं सुदूर पृथ्वी में जब हमारे रोम अन्तिम
मिले होंगे
तब अचानक हिली होंगी फुनगियाँ तक
एक ही तो मूल में हम
एक ही है भूमि-जल-संसार अपना
एक ही अन्तःप्रदेश-
उतने ही अलग
उतने ही एक
जितना दो आँखें ।" [5]

जगत के देशों के बीच सशस्त्रीकरण की प्रतिस्पर्धा जारी है । एक ओर निःशस्त्रीकरण का वाद-विवाद भी प्रचलित है । विश्व के बुद्धिजीवी मानते हैं कि आल्बर्ट आइन्स्टीन के मुताबिक तीसरा विश्व युद्ध संसार का सर्वनाश कर देगा । इसका मूलकारण परमाणु-सशस्त्रीकरण है । हालांकि परमाणुविक निःशस्त्रीकरण की नीति से विश्वराजनेतागण सहमत हैं, फिर भी वे परमाणुविक सशस्त्र राष्ट्र घोषित करवाने में गर्व करते हैं। यह सत्य है कि परमाणुविक सशस्त्र राष्ट्र भी विश्वयुद्ध का शिकार होगा । फिर क्यों यह होडाहोडी है । कवि अरुणकमल अपनी ‘ईर्ष्या' नामक कविता में इस बात को दर्शाति हैं कि विश्व की शक्तिशाली व परमाणु-अस्त्रसंपत्र राष्ट्र विश्वजीत के सपने में कहीं खुद को भी न विनष्ट होने दे । कवि कहना चाहते हैं कि जो दूर के लघु राष्ट्र हैं वे परमाणु-बम के विस्फोट के विकरणों से फ्रिडज के फ्रीजर के विरचित वातावरण में छिपकर शायद गंभीर घायल होकर भी बच सकेंगे, लेकिन वैसे अवसर महान एवं विकसित देशों को नहीं हैं। कवि इसी बात को व्यंग्य से कहना चाहते हैं ।

$$
\text { “सचमुच विश्वजीत }
$$

मुझे तुम्हारा यह ऐश ट्रे बहुत पसंद है

बिल्कुल पॉपी के फूल की तरह 
खिल रहा तुम्हारे टेबुल पर सचमुच

कल न्यूँट्रन बम गिरेगा

हम तुम सब मर जाएँगे

सब कुछ नष्ट हो जाएगा

फिर भी इस टेबुल पर इसी तरह चमकता रहेगा शान से यह ऐश ट्रे ।" [6]

विकसित देशों में नदियाँ सर्वाधिक खतरे में हैं। एक वैश्विक अध्ययन से पता चला है कि नदियों के प्राकृतिक प्रवाह में आ रहे लगातार अवरोधों के कारण नदियों और उसके सहारे चल रहा जीवन और जैव विविधता सभी कुछ संकट में पड़ गए हैं। आवश्यकता इस बात की है कि हम पुनः नदियों के प्राकृतिक प्रवाह को सुनिश्चित करें। सिर्फ मनुष्यों के लिए पानी की उपलब्धता सुनिश्चित कराने हेतु नदियों के प्रवाह पर लगती रोक अंततः विनाशकारी सिद्ध होगी। विश्व की नदियाँ कुप्रबंधन और प्रदूषण से संकट में हैं। वैज्ञानिकों ने खनन, प्रदूषण, सघन खेती, केचमेंट क्षेत्र में अवरोध और बांध निर्माण जैसे प्रभावों की पहचान करके नदियों के स्वास्थ्य और उससे जुड़े रहवास पर पड रहे प्रभाव दर्शाये हैं। समुद्र में सामूहिक रूप से आधे से अधिक अपवाह यानी पानी पहुंचाने वाली सबसे बड़ी अमेज़ान, अमूर, चांग जियांग, ब्रहमपुत्र जैसी सैंतालीस नदी प्रणालियों में से तीस खतरे में हैं। अमेरिका और पश्चिमी यूरोप की अधिकांश नदी प्रणालियां सर्वाधिक खतरे में हैं। नदियों में होनेवाले ह्रास पर कवि ने अपनी 'शोक' नामक कविता में इस प्रकार कहा है।

“इस अंचल की एक इंच भूमि भी

ऐसी नहीं कि जहाँ होकर गुजरी न हो

यह नदी

पर क्रोध नहीं शोक है मुझे कि

बार बार जो बदलती रही रास्ता

बार बार जो पोंछती रही अपने ही छाप

जो एक पल कभी बैठी नहीं थिर

पा न सकी वो रास्ता अब तक

जिसे ढूँढ़ती फिरी सारी धरती उकटेर ।" [7]

विश्व की रीति-नीति सब शासकों व राजनेताओं की कृति है । विश्वराजनेता अपने-अपने देश के किसान, मजदूर, कर्मचारीवर्ग और अन्य निम्न मध्यवर्गीय लोगों को यह आशा व वादा देकर सत्तारूढ हो जाते हैं कि उनकी आर्थिक स्थिति व स्तर में विकास होगा । लेकिन असलियत में बिलकुल आमजनता विरुद्ध शासनतंत्र ही लोकतंत्र के नाम पर जारी है । वे अमीर, पूँजीपति, उच्चवर्ग आदि के पक्षधर होकर नजर आते हैं । अमीर और अमीर बनते जा रहे हैं और गरीब कंगाल बनकर दारुण अवस्था में ही पडे हैं । यह अनादि काल का सवाल है कि महान चिंतक, दार्शनिक, बुद्धिजीवी, शुभचिंतक जैसे महानुभवों के रहते विश्व में उनसे सम्मत व निर्मित शासनतंत्र व तत्संबंधी प्रक्रियाओं के बावजूद हर राष्ट्र की परिस्थिति ऐसी क्यों है। कवि अरुणकमल
जी अपनी 'पुराना सवाल' शीर्षक कविता में यही प्रश्न पेशकर उत्तेजित होते हैं।

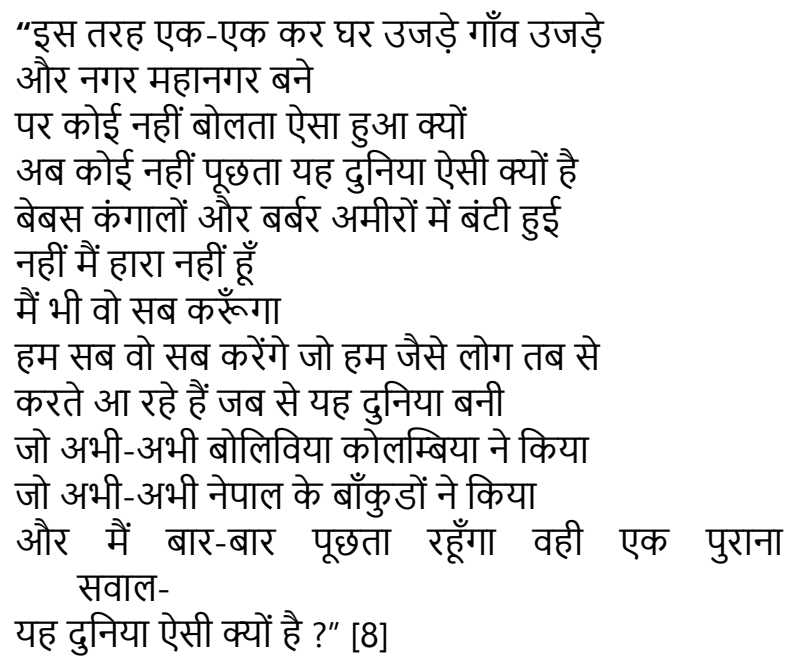

शिक्षा संपन्न कर्मचारी वर्ग अवगत हैं कि विश्व की राजनीति पूँजीपति की है । उनकी जागरूकता से जगत का समाज क्रांति की गतिविधि से परिवर्तित होने की दिशा में अग्रसर होना चाहता है। आजकल किसान, मजदूर भी शासन विधान से वाकिफ हैं। इसलिए कवि पुनर्जगृत करना चाहते हैं कि साम्यवादी हृष्टिकोण से सब आक्रोश व उत्तेजना से इस जगत को घेर लें तब तक जब तक शासन नीतियों में परिवर्तन न किया जाए। उन्होंने अपनी कविता ‘एक पुराना गान' में लिखा है ।

\author{
“सब कुछ खो तो नहीं गया \\ अभी भी क्यूबा है दूब सा खड़ा झंझावात में \\ अभी भी वियतनाम और चीन में झंडा है लाल \\ अभी भी हमें वे गीत सब याद हैं \\ जो स्पार्टकस ने गाए जो पोरिस कम्यून से लेकर \\ शिकागो मजदूरों सोवियतों पुत्रप्रावायलार \\ सन्तालों ने गाए \\ फिर से उठी है हवा दूर वेनजुएला से \\ माच्चू पिच्चू के शिखरों से फिर सगरमाथा \\ हिमाला से \\ वे बीज जो छींटे गए पूरी पृथ्वी पर ।" [9]
}

जगत के विकसित देशों की नीति चली है कि विकासेच्छुक कम प्रजावाले राष्ट्रों में दखलंदाजी राजनीति करें। विशेषतः उन देशों में जहाँ अपुनर्नवीकरणीय इंधन स्रोत उपलब्ध हैं, वहाँ तो जोरों पर ऐसी हस्तक्षेपी राजनीति चलती आ रही है। रणनीति प्रशिक्षण, आतंकवादविरोधी प्रशिक्षण या फौजी सहारा ऐसे वादों से उन सांराज्यों को घेर लेते हैं । यह अन्यायी व अत्याचारी प्रवृत्ति है। ट्यूनीशिया व लीबिया जैसे देशों में तो सरकार का शासक नाममात्र रह गए हैं या किसी को नहीं पता शासन का बागडोर किसके हाथ में है। विडम्बनात्मक स्थिति है कि बस देशी शासन 
रहा है, पर विदेशी सेना। ऐसी अन्तर्राष्ट्रीय राजनीति से सचेत होकर अपनी कविता ‘हवा में हाथ’ में दर्शाते हैं।

“क्या हुक्मरान सचमुच भाग जाएँगे

ट्यूनीशिया लीबिया बहरैन से

क्या सचमुच वहाँ ऐसी आजादी होगी

कि लोग खुद अपनी सरकार चुन और चला सकें

या फिर जिएँ बिना सरकार के ?

और हुक्म बस हवा का चले या धूप और चाँद का

पर फल खजूर के अपनी मर्जी से पकें और झरें

या फिर वैसा ही होगा जैसा इराक

अफगानिस्तान में

कि हुक्म तो देशी हो पर फौजें विदेशी

और दिन और रात बराबर हों

अवाम की जिन्दगी में ?" [10]

\section{4. निष्कर्ष}

कवि अरुणकमल जी प्रगतिवादी एवं प्रगतिशील हष्टिकोणों से देश-विदेश की घटनाओं व परिस्थितियों को अपनी कविताओं का विषय बनाया है । उनकी कविताओं में अंतरराष्ट्रीय चेतना सार्वभौमिक बुद्धिविचार के धरातल पर दृष्टिगत होती है । उनकी विदेशी समस्याओं को देखने-परखने की विचारधारा प्रशंसनीय है । यह उनकी विश्वप्रजापतित्व भावना की प्रतीक है । अविकसित और अर्धविकसित लघु राष्ट्रों के प्रति उनकी संवेदनशीलता भावविभोर प्रयास है । ट्यूनिशिया, लीबिया, काम्भोज आदि लघु देशों की देश-कालीन परिस्थितियों से वाकिफ होकर अपना विचार कविताओं द्वारा प्रकट करने की चेष्टा उनकी समानुभूति का परिचायक है । जगत की ज्वलंत समस्याओं को लेकर कविता-सृजन उनकी जागरूकता और उनकी विश्वविकास की चेतना को उजागर करता है । विश्व अपमिश्रण की समस्या, विश्व शासनतंत्र की नीति, विश्व नदी-समस्या, विश्व मेहनतकश की समस्या, विश्व कर्मचारी-शोषणतंत्र, विश्वसशस्त्रीकरणनिःशस्त्रीकरण का मुद्दा, उनके ग्लोबल विल्लेज का विचार, वियतनाम व काम्भोज के आंतरिक युद्ध व तत्संबंधी सिलसिला आदि अंतरराष्ट्रीय पहलुओं के प्रति कवि अरुणकमल जी संवेदनशील हैं और सार्वभौमिक अवगमन की क्षमता और सार्वभौमिक स्वभागिता धरकर विश्वप्रगतिशील हैं। उनके कविताओं में विश्वचेतना व अंतरराष्ट्रीय चेतना परिलक्षित हैं ।

\section{संदर्भ}

[1] आचार्य रामचंद्र वर्मा, मानक हिन्दी कोश, प्रयाग

[2] अरुणकमल, अपनी केवल धार, पू.11, वाणी प्रकाशन, नई दिल्ली

[3] अरुणकमल, अपनी केवल धार, पृ.19, वाणी प्रकाशन, नई दिल्ली

[4] अरुणकमल, अपनी केवल धार, पृ.19, वाणी प्रकाशन, नई दिल्ली

[5] अरुणकमल, अपनी केवल धार, पृ.26, वाणी प्रकाशन, नई दिल्ली

[6] अरुणकमल, अपनी केवल धार, पृ.52, वाणी प्रकाशन, नई दिल्ली

[7] अरुणकमल, नए इलाके में, पृ.81, वाणी प्रकाशन, नई दिल्ली

[8] अरुणकमल, मैं वो शंख महाशंख, पृ.56, वाणी प्रकाशन, नई दिल्ली
[9] अरुणकमल, मैं वो शंख महाशंख, पृ.57, अरुणकमल, मैं वो शंख महाशंख, पृ.78, वाणी प्रकाशन, नई दिल्ली

[10] अरुणकमल, मैं वो शंख महाशंख, पृ.78, वाणी प्रकाशन, नई दिल्ली अंतरजाल-चेतना के पाँच आयाम-

[11] अंतरजाल-https://hi.wikipedia.org/wiki/चेतना

[12] अंतरजाल-चेतना के पाँच आयाम-

[13] http://literature.awgp.org/book/Gayatree_kee_panchakoshee_sadhan $\mathrm{a} / \mathrm{v} 7.13$

[14] अंतरजाल -CWLG Definition - Global Consciousness https://www.surveymonkey.com/r/ZYJ7CW3

[15] अंतरजाल-https://libguides.westsounda cademy.org/wsee/global-consciousness

[16] अंतरजाल- https://hi.wikipedia.org/wiki/मिलावट 